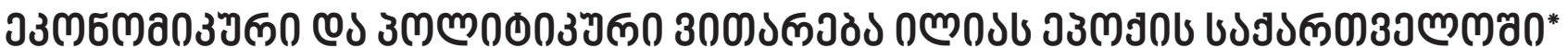

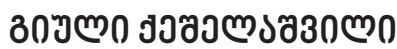

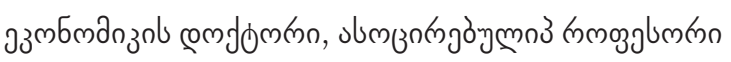

https://doi.org/10.35945/gb.2018.06.018

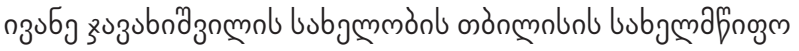

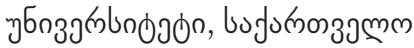

giuli.keshelashvili@tsu.ge

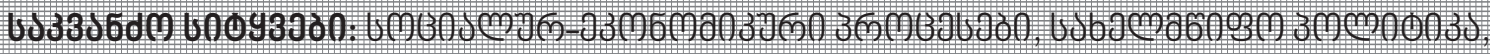

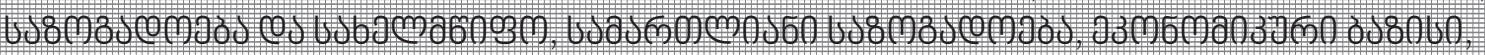

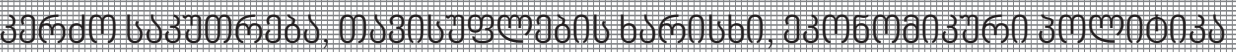

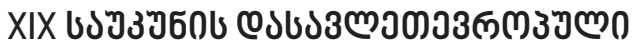

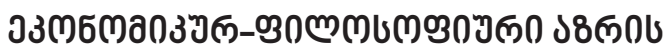

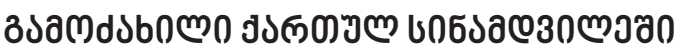

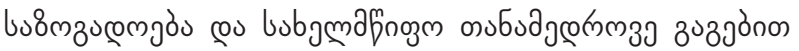

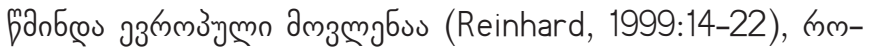

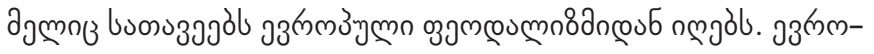

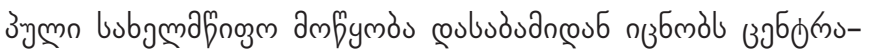

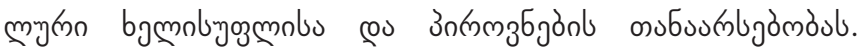
nbom

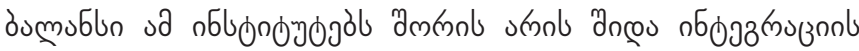

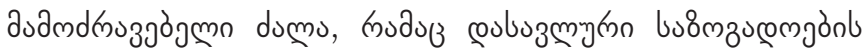

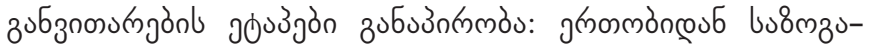

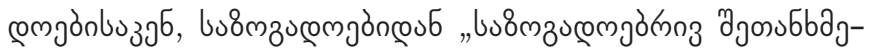

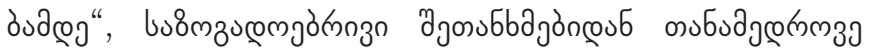

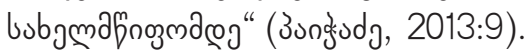

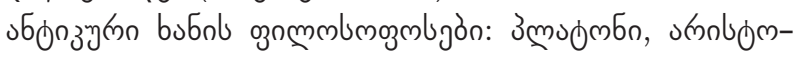

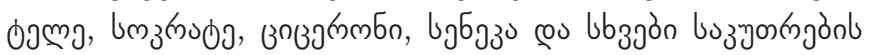

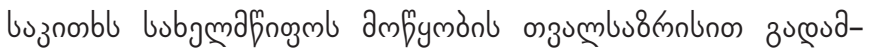

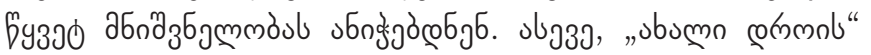

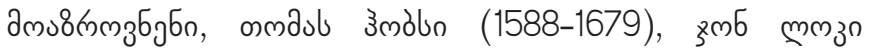

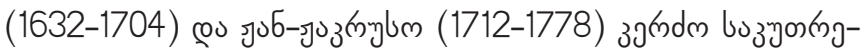

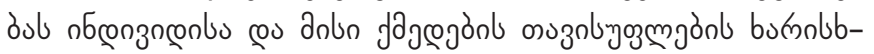

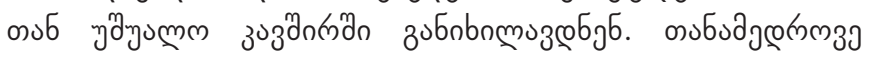

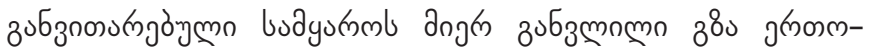

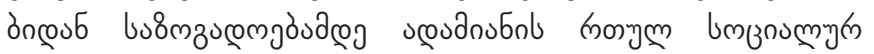

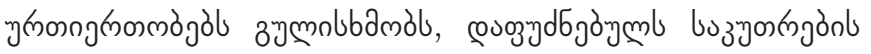

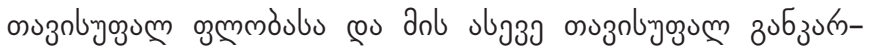

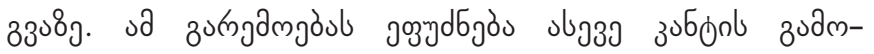

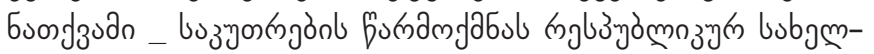

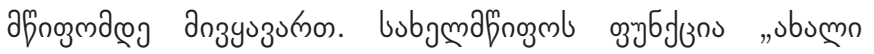

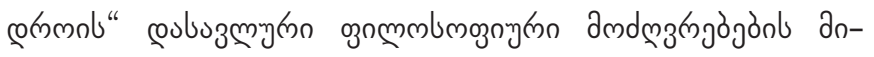

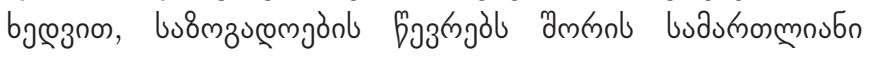

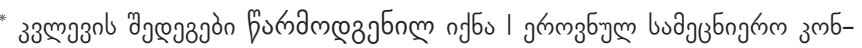

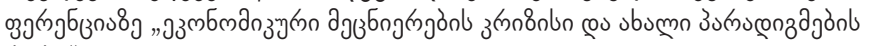
dngos"

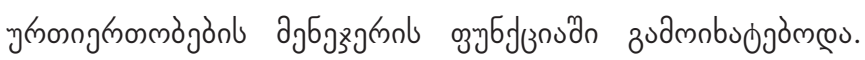

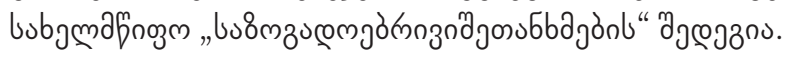

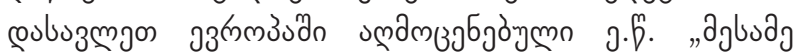

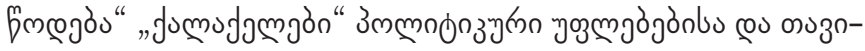

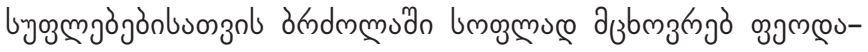

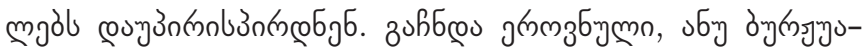

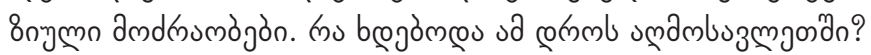

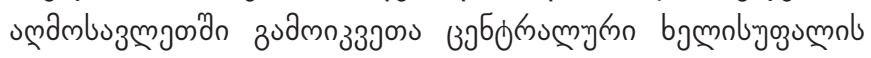

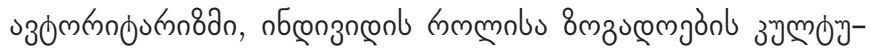

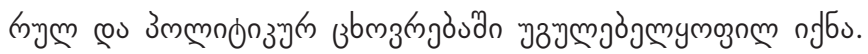

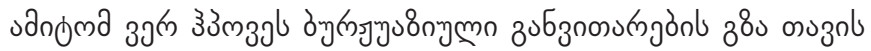

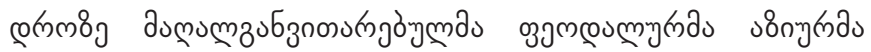

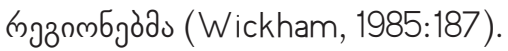

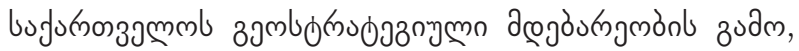

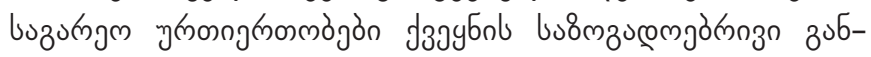

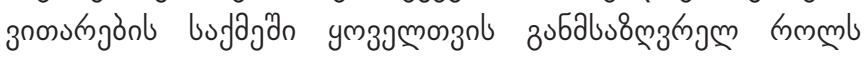

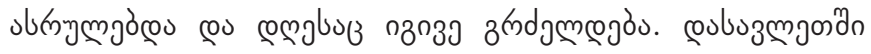

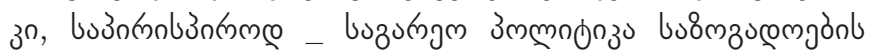

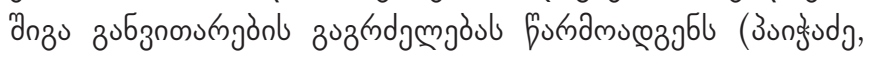

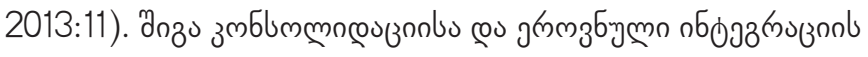

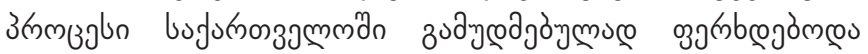

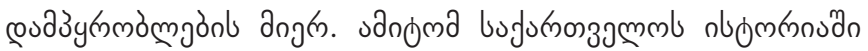

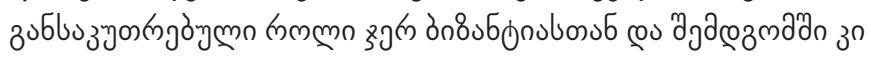

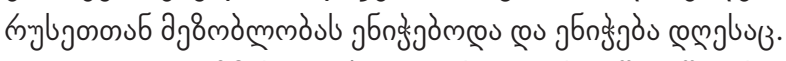

øgm

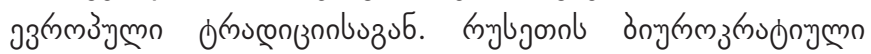

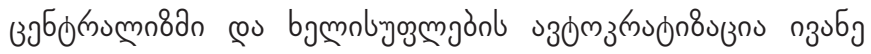

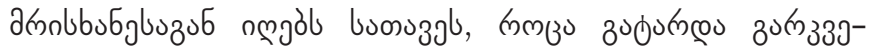

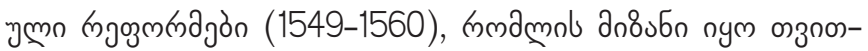

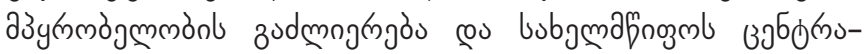
mnosuznol zuбa

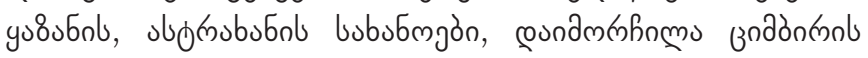

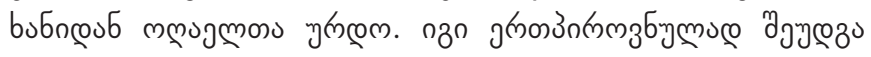

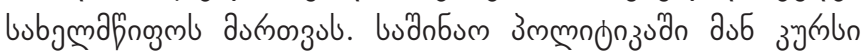

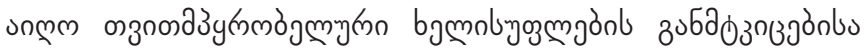

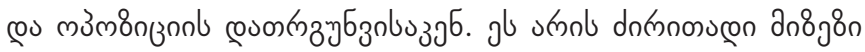

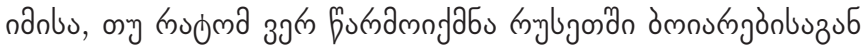




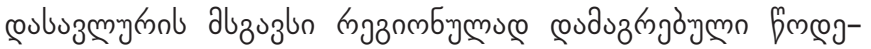

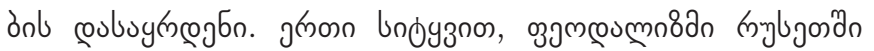

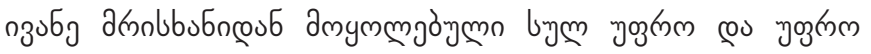

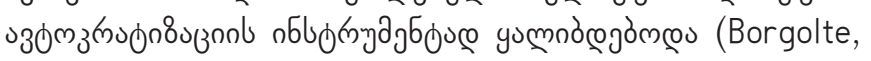
$2002: 268)$.

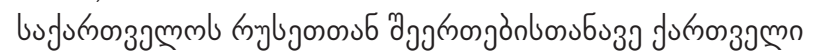

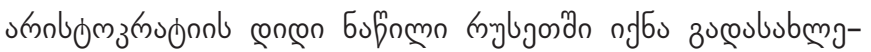

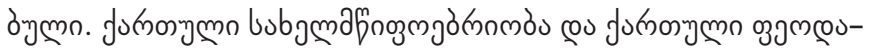

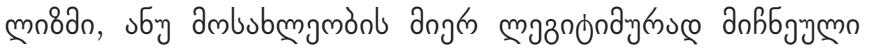

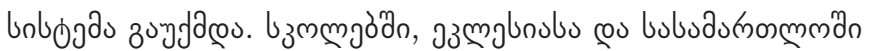

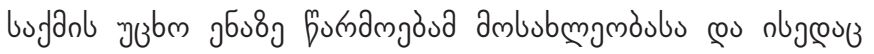

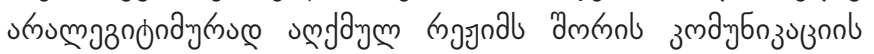

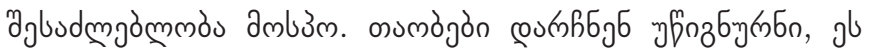

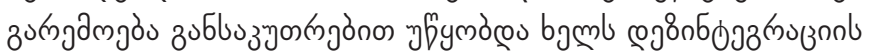

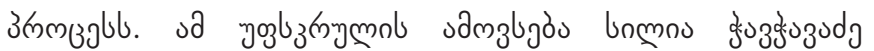

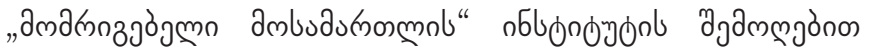
उзоnmmónou.

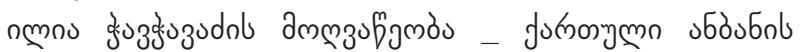

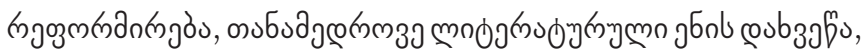

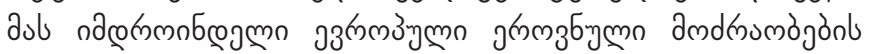

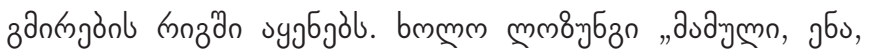

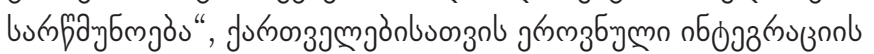

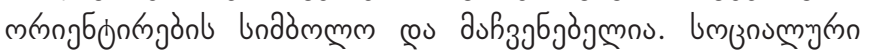

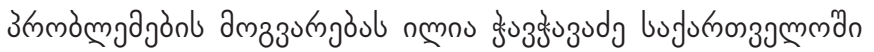

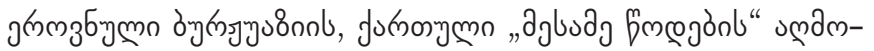

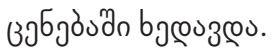

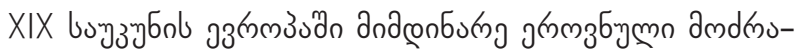
màjòn grmm

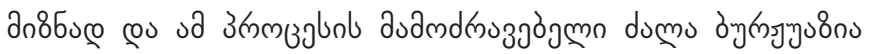

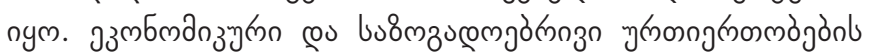

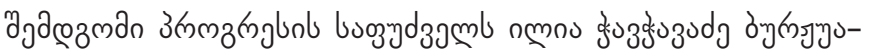

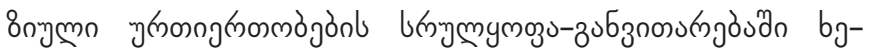

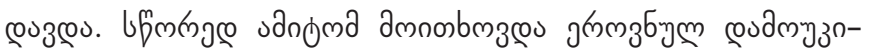

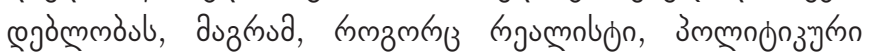

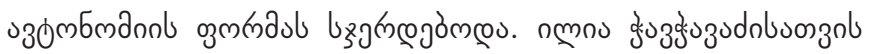

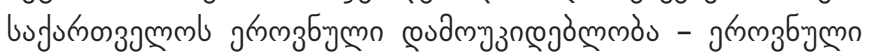

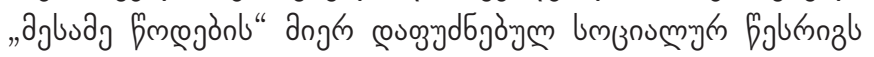

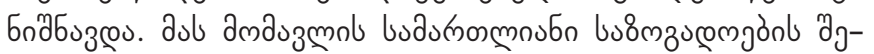

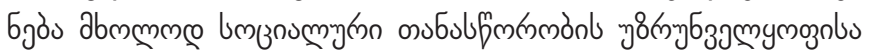

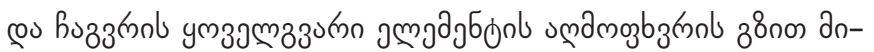

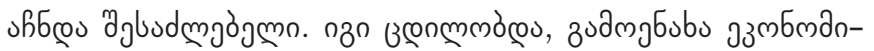

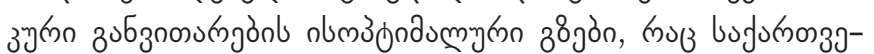

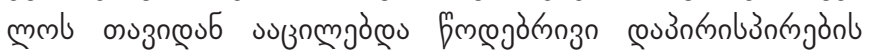

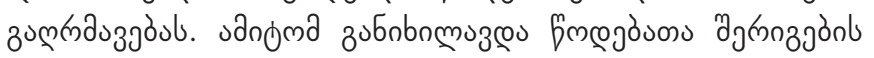

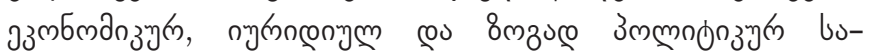

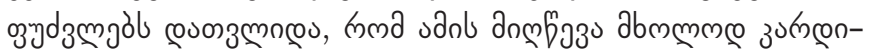

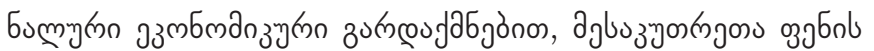

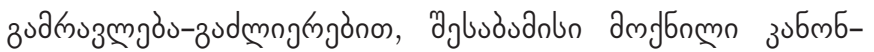

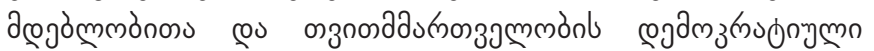

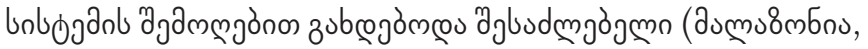
1999:112).

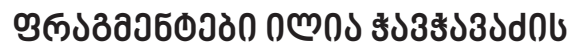

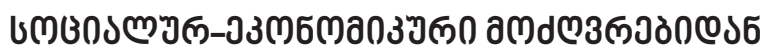

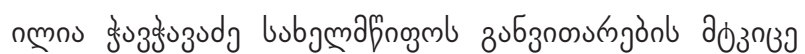

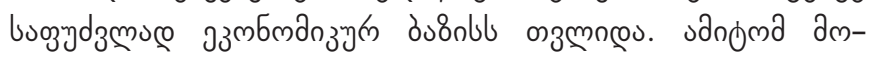

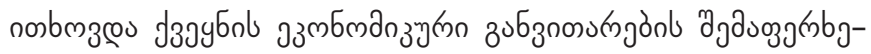

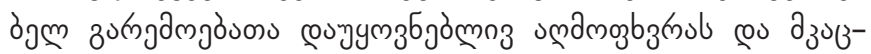

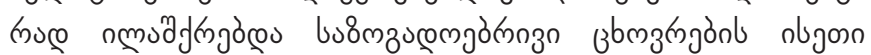

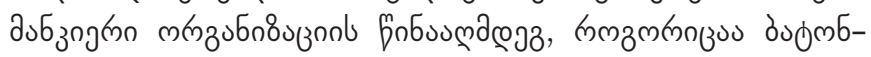

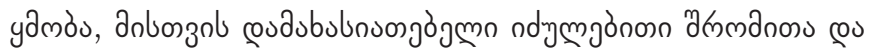

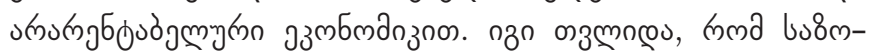

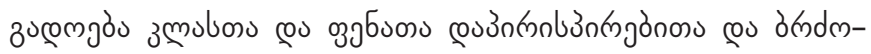

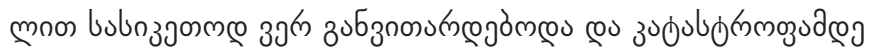

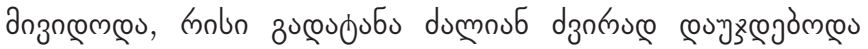

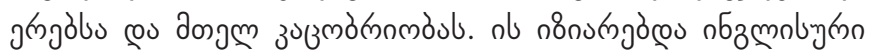

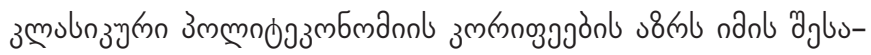

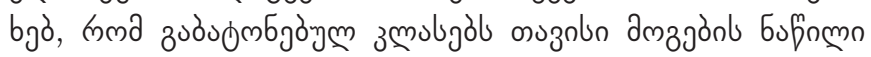

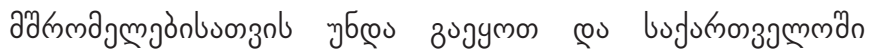

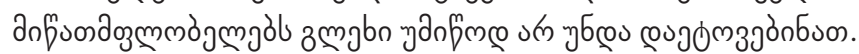

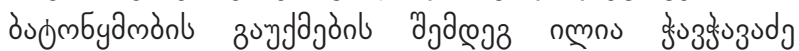

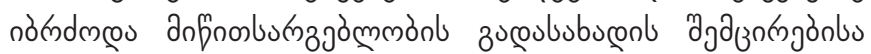

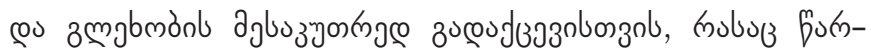

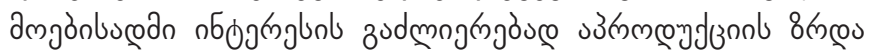

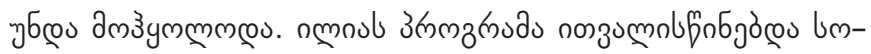

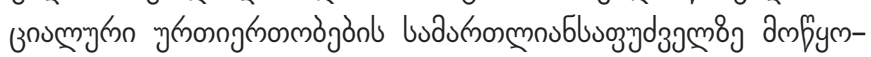

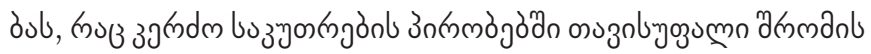

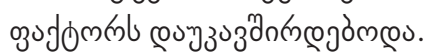

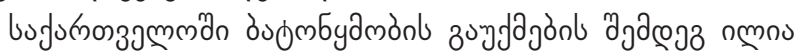

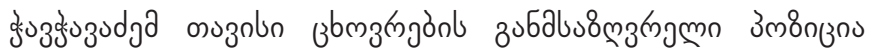

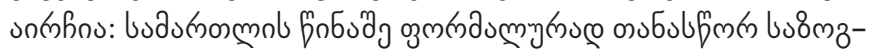

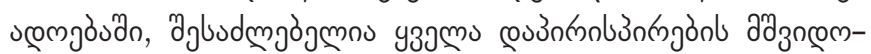

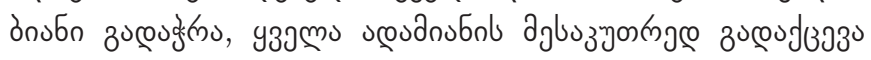

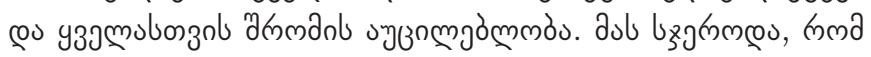

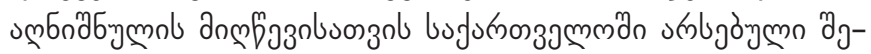

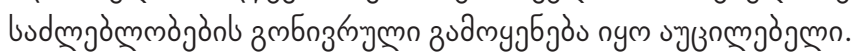

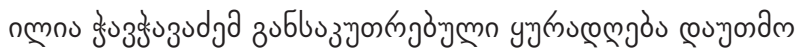

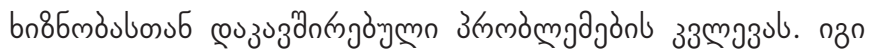

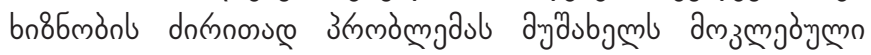

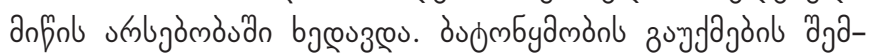

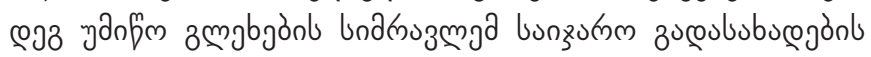

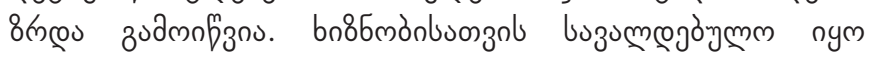

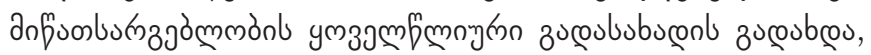

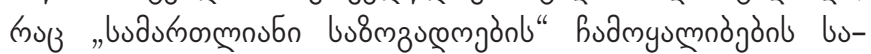

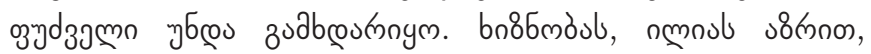

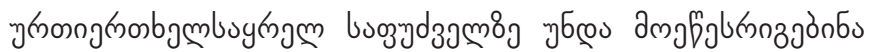

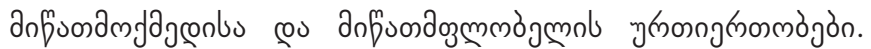




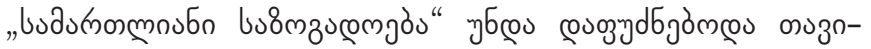

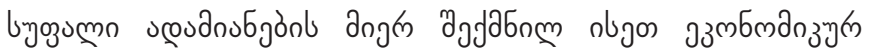

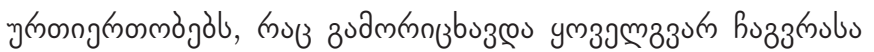

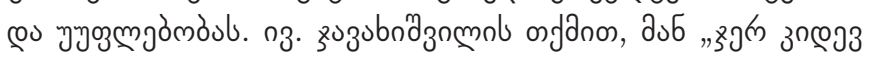

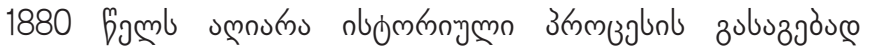

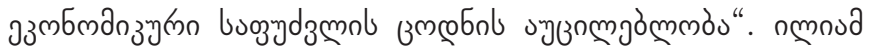

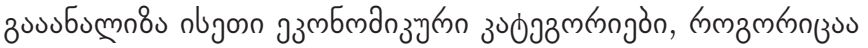

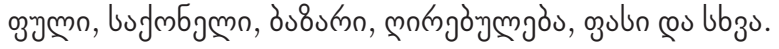

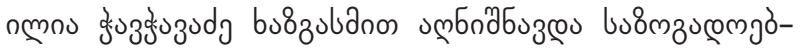

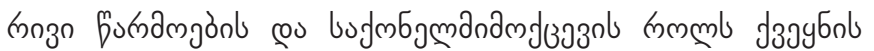

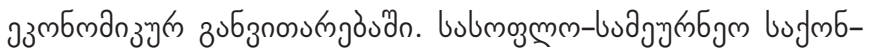

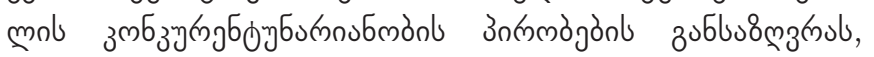

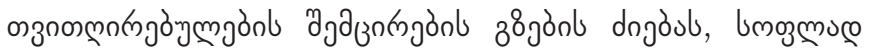

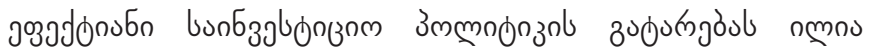

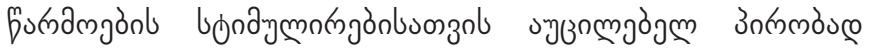

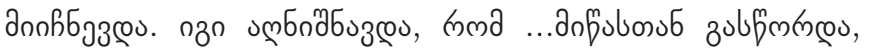

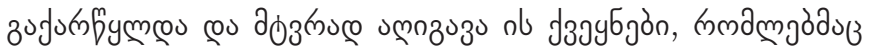

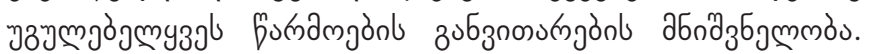

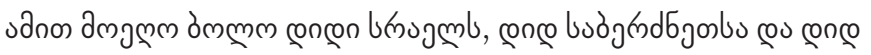

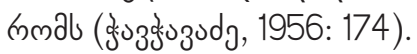

"buonuzu@os

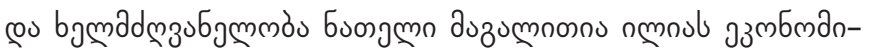

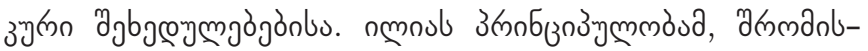

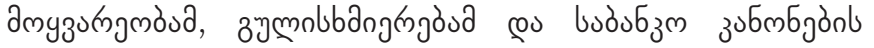

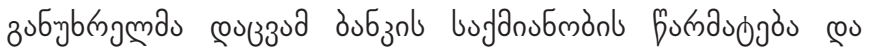

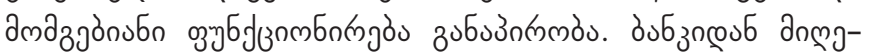

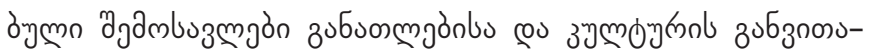

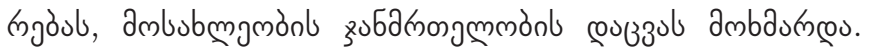

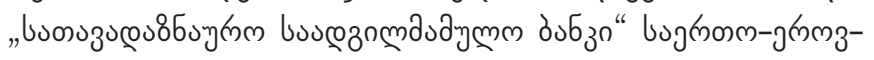

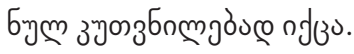

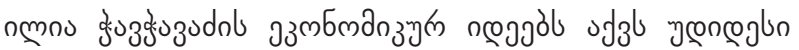

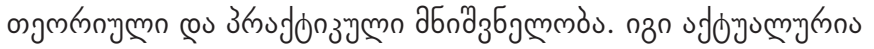

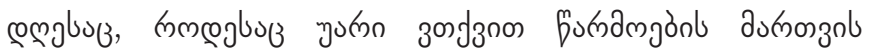

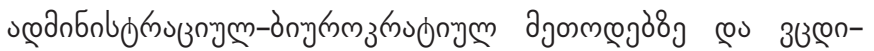

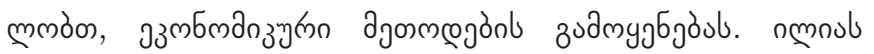

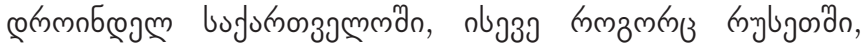

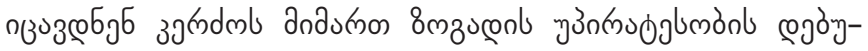

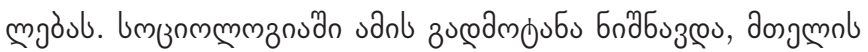

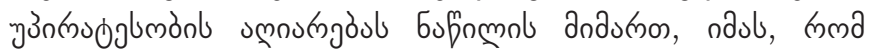

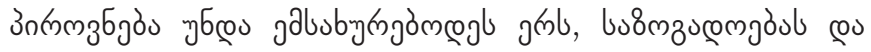

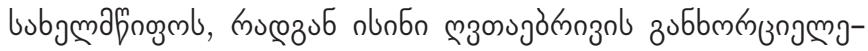

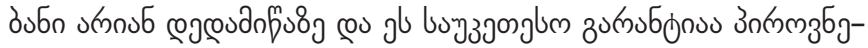

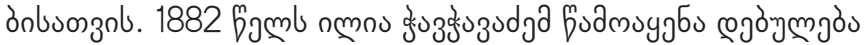

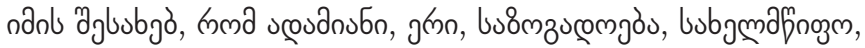

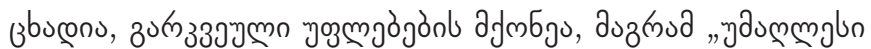

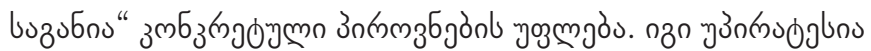

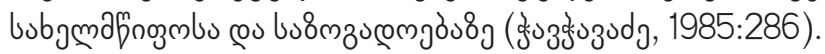

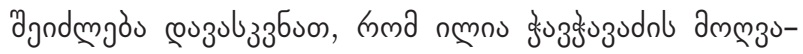
fymòs ry

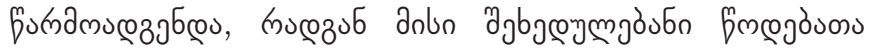
चgm

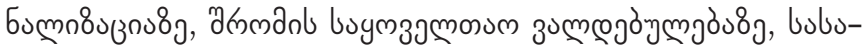

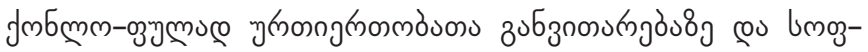

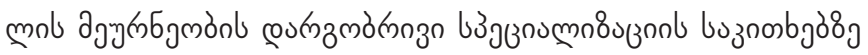

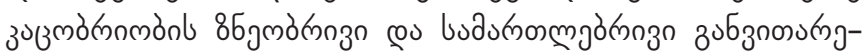

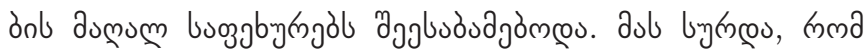

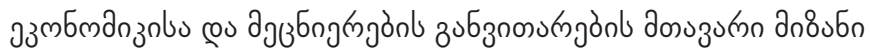

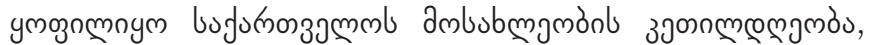

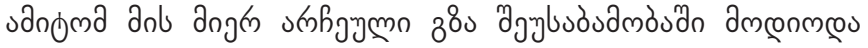

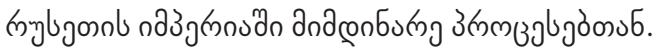

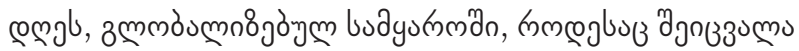

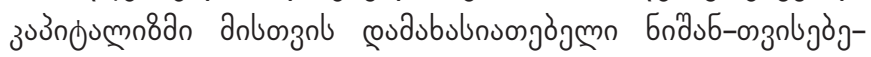

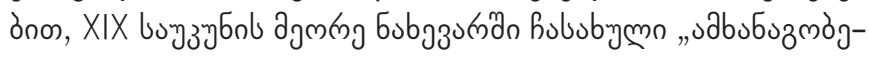

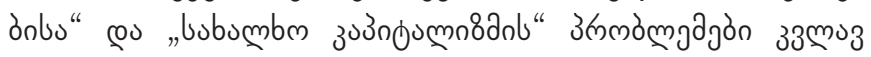

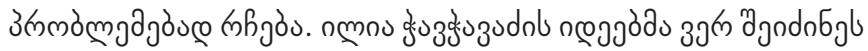

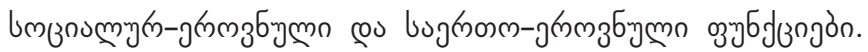

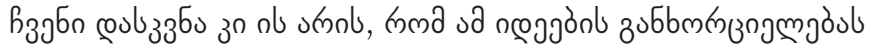

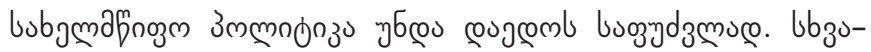

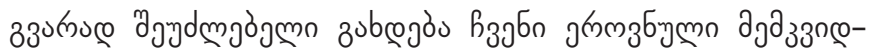

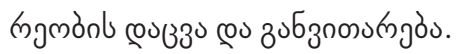




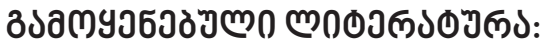

1. Reinhard W., (1999). Geschichte der Staatgewalt. Eine vergleichende Verfassungsgeschichte Europas von den Anfängen bis zur Gegenwart, Oskar Beck, München.

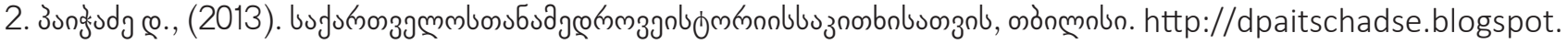
com/2013/04/blog-post_6115.html

3. Wickham Ch., (1985). The Uniqueness of the East, krebulSi: Feudalism and Non-European Societies, T.J. Byres und H. Mukhia (Hrg), London.

4. Borgolte M., (2002). Otto Hinzes Lehre vom Feudalismus in kritischen Perspektiven des 20. Jahrhunderts, krebulSi: Nathaly Fryde, Pierre Monnet, Otto Gerhard Oexle (Hrg), Die Gegenwart des Feudalismus, Göttingen.

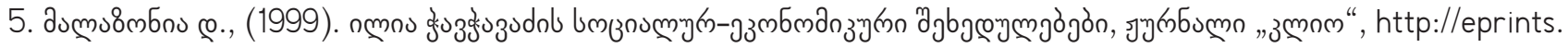
iliauni.edu.ge/usr/share/eprints3/data/2125/1/klio-\%203.pdf

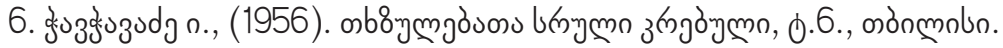

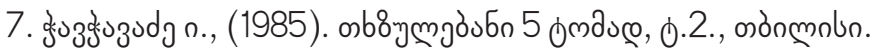

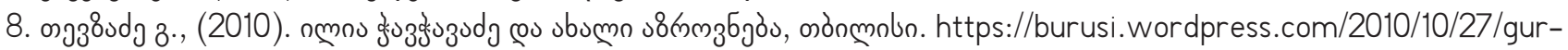
am-tevzadze

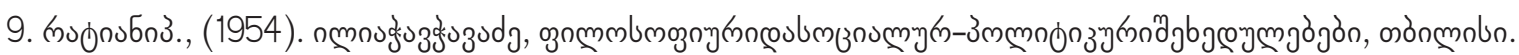

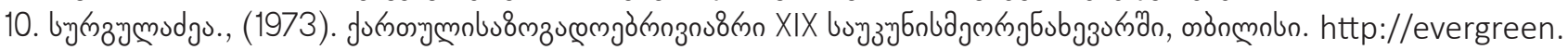
tsu.ge/eg/opac/advanced?locg=1

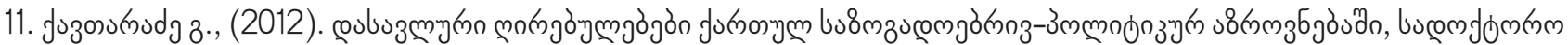

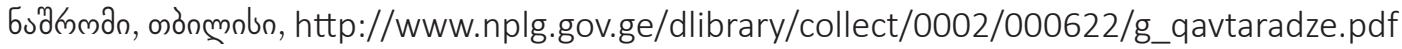




\section{ECONOMIC AND POLITICAL SITUATION IN THE ILIA ERA IN GEORGIA}

\section{GIULI KESHELASHVILI}

https://doi.org/10.35945/gb.2018.06.018

Doctor of Economics, Associated Professor

Ivane. Javakhishvili Tbilisi State University, Georgia

Giuli.keshelashvili@tsu.ge

\section{KEYWORDS: SOCIO-ECONOMIC PROCESSES, STATE POLICY, SOCIETY AND STATE, FAIR SOCIETY,}

ECONOMIC BASE, PRIVATE PROPERTY, QUALITY OF FREEDOM, ECONOMIC POLICY

\section{SUMMARY}

The article offers a discussion of the socioeconomic processes in Georgia in the second half of $19^{\text {th }}$ century characterized with significant changes in socioeconomic, political and cultural life of Georgian people. In the mentioned period, capitalistic production relations emerged earlier have developed and this was followed by fundamental changes in balance of power between classes, public thinking and sphere of material and mental life. Due to geo-strategic location of Georgia, the foreign relations were decisive for the social development of the country. Historically, the process of internal consolidation and national integration has permanently been delayed by the conquerors. Neighborhood relations with Russia have played the particular role in the foreign policies of Georgia.

Work focuses on Ilia Chavchavadze's views about the role of production and goods' circulation with respect of country's economic development, issues of improvement of agricultural products' competitiveness, implementation of effective investment policies in rural areas, establishment and development of the "banks of the nobility".

Irrespective of thorough study of these problems, the above issues are still of significance today. The work demonstrates that Ilia Chavchavadze's activities were particularly dangerous for Russian Empire. His views about conciliation between different titles, rationalization of the landlords and peasant farm economies, general labor obligations, commercialization and agriculture specialization corresponded to the high level of the moral and legal development of the mankind. Ilia desired that the main goal of the development of economy and science was welfare of Georgian population and therefore, the way chosen by him contradicted to the processes in Russian Empire. In the end of the work the conclusion is made that today, with change of capitalism, with its characteristic features, the problems of "partnerships" and "public capitalism" emerged in the second half of $19^{\text {th }}$ century still persist. Ilia Chavchavadze's ideas could not acquire the social-national and general-national functions as implementation of these ideas should be based on the state politics. Otherwise, our national heritage cannot be protected and developed. 